\title{
Room temperature single-photon sources based on single colloidal nanocrystals in microcavities
}

\author{
Antonio Qualtieri ${ }^{\mathrm{a}, *}$, Giovanni Morello $^{\mathrm{a}}$, Piernicola Spinicelli ${ }^{\mathrm{b}}$, \\ Maria T. Todaro ${ }^{\mathrm{a}}$, Tiziana Stomeo ${ }^{\mathrm{a}}$, Luigi Martiradonna ${ }^{\mathrm{a}}$, Milena De Giorni ${ }^{\mathrm{a}}$, \\ Xavier Quélin ${ }^{c}$, Stéphanie Buil ${ }^{c}$, Alberto Bramati ${ }^{\mathrm{b}}$, Jean P. Hermier ${ }^{\mathrm{b}, \mathrm{c}}$, \\ Roberto Cingolani ${ }^{\mathrm{a}}$, Massimo De Vittorio ${ }^{\mathrm{a}}$ \\ ${ }^{a}$ National Nanotechnology Laboratory (NNL) of CNR-INFM, Distretto Tecnologico ISUFI, Università del Salento, via per Arnesano, \\ 73100 Lecce, Italy \\ b Laboratoire KASTLER BROSSEL - Université Paris 6, Ecole Normale Supérieure et CNRS, UPMC case 74, 4 Place Jussieu, 75252 Paris \\ Cedex 05, France \\ ${ }^{\mathrm{c}}$ Groupe d'étude de la Matière Condensée, CNRS UMR8635, Université de Versailles Saint Quentin, 45 avenue des Etats-Unis, 78035 \\ Versailles Cedex, France
}

\section{A R T I C L E I N F O}

\section{Article history:}

Available online 21 June 2009

\section{Keywords:}

Colloidal nanocrystals

Electron beam lithography

Single-photon device

Microcavity

\begin{abstract}
A B S T R A C T
Direct lithography of resist blends, embedding semiconductor colloidal nanocrystals (NCs) is an innovative way to achieve nanopositioning of NCs in quantum-confined optical resonators. In this work, we show a new appealing approach for the fabrication of single-photon sources operating at room temperature by localizing semiconductor colloidal NCs into vertical planar microcavities with lithographic techniques.
\end{abstract}

(C) 2009 Elsevier Ltd. All rights reserved.

\section{Introduction}

The development of unconditionally secure quantum communication systems is still hindered by the limited availability of cheap and bright Single-Photon Sources (SPS) operating at room temperature. Among the proposed sources of single photons [1-3], epitaxially grown quantum dots (QDs) have had particularly major efforts addressed to them [4,5], although the maximum operating temperature for epitaxial QD-based SPS is still far from ambient conditions [6]. Wet-chemistry-based colloidal nanocrystals (NCs) as quantum-confined emitters for photonic devices have recently [7-9] been considered promising alternatives due to their lower fabrication costs, high quantum efficiency

\footnotetext{
* Corresponding author. Tel.: +39 0832 298200; fax: +39 0832298386.

E-mail address: antonio.qualtieri@unile.it (A. Qualtieri).
} 


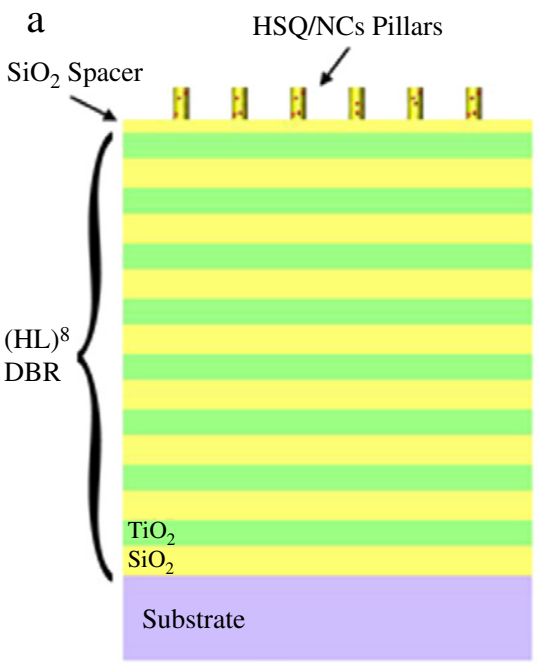

b

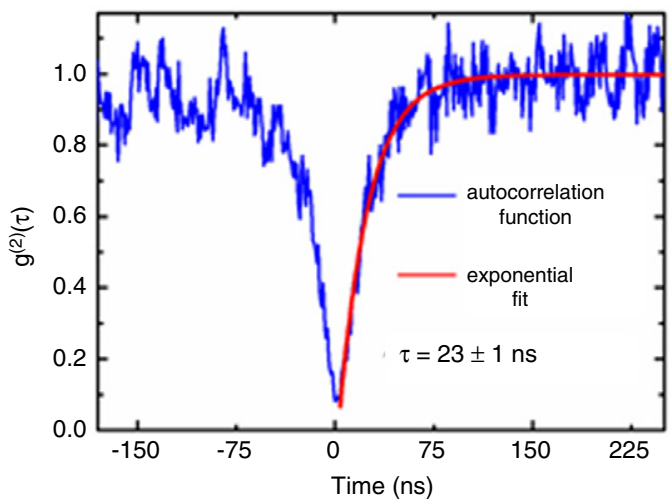

Fig. 1. (a) Sketch drawing of an array of pillars, embedding $\mathrm{CdSe} / \mathrm{ZnS}$ quantum dots, localized on the top of a $\mathrm{SiO}_{2} / \mathrm{TiO}_{2} \mathrm{Bragg}$ mirror and of an additional $\mathrm{SiO}_{2}$ spacing layer; (b) anti-bunching profile of single-QD fluorescence under pulsed excitation. The red solid line is a fit yielding a $23 \mathrm{~ns}$ excited-state lifetime for a nanocrystal inside the pillar. (For interpretation of the references to colour in this figure legend, the reader is referred to the web version of this article.)

at room temperature, high versatility in the chemical synthesis and broad tunable emission range (from visible to IR) [10,11]. Moreover, several groups are proposing different approaches [12-14] for controlling the peculiar spectral features (such as blinking, spectral diffusion and long luminescence lifetimes $[15,16]$ ) which have prevented the diffusion of SPS based on colloidal NCs so far. In particular, the insertion of emitters in quantum-confined optical resonators $[17,18]$ could be an effective way to engineer their radiative decay and to increase the collection efficiency of single photons. Here we develop a new approach for the fabrication of SPS operating at room temperature by localizing semiconductor colloidal NCs into vertical planar microcavities with lithographic techniques.

\section{Experimental}

A distributed Bragg reflector (DBR) consisting of eight pairs of $\mathrm{SiO}_{2} / \mathrm{TiO}_{2}$ was grown by electron beam evaporation on a silicon substrate and an additional $\mathrm{SiO}_{2}$ spacing layer was deposited on top of it (Fig. 1(a)). The design of the distributed Bragg reflectors and of the microcavity was performed through simulations based on the transfer matrix method, in order to tune the resonance cavity at the central emission wavelength of the colloidal nanocrystals. Finite-Difference Time-Domain (FDTD) calculations also confirmed the spatial distribution of the resonant mode in the cavity.

A very low concentration of CdSe/ZnS colloidal nanocrystals emitting at $\lambda=615 \mathrm{~nm}$ was dispersed in a high resolution electron beam resist, namely Hydrogen SilsesQuioxane (HSQ) [19]. As previously reported [20], nanocrystals embedded in electronic resists can be easily patterned by means of traditional lithographic processes, since the presence of semiconductor clusters in the matrix does not affect the sensitivity of the polymeric host significantly and, at the same time, the emission properties of the nanocrystals are not damaged by the interaction with the electron beam. A $90 \mathrm{~nm}$ thick layer of the blend was spin-coated on the bottom structure and subsequently exposed to the electron beam (acceleration voltage $=20 \mathrm{kV}$, beam current $=14 \mathrm{pA}$ ) to define an array of active pillars with diameters ranging from $500 \mathrm{~nm}$ down to $30 \mathrm{~nm}$.

As described by Purcell et al. [17], the enclosure of an emitting material into an optical cavity which alters the distribution of photonic modes is an efficient way to engineer the radiative lifetime of the emitter itself. We therefore realized a complete microcavity starting from the previous structure. The array of pillars was planarized by a pure HSQ layer; the planarization of the central cavity helps in 


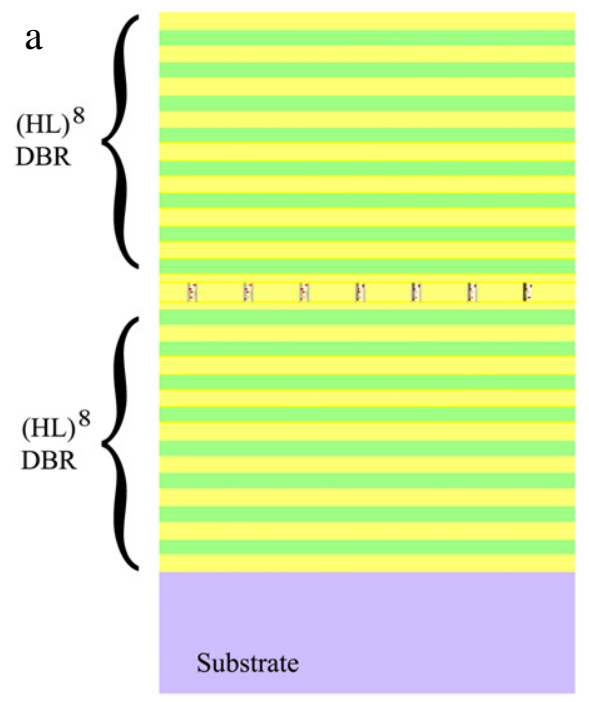

$\mathrm{b}$

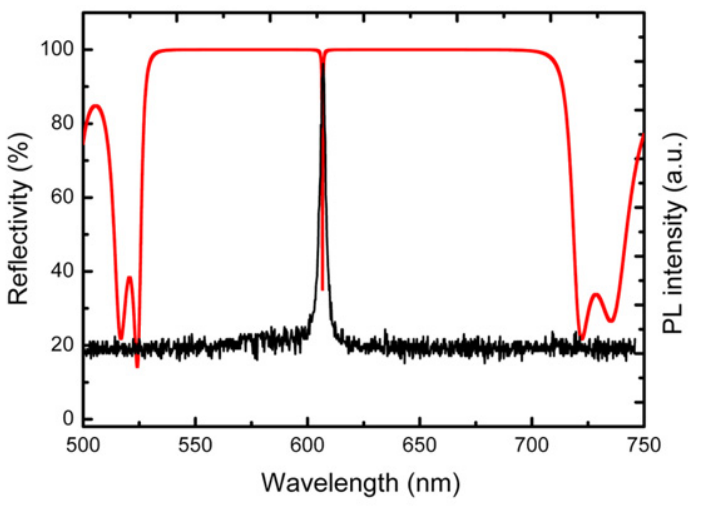

Fig. 2. (a) Schematic drawing of the $\lambda / 2$ planar cavity consisting of two $\mathrm{SiO}_{2} / \mathrm{TiO}_{2}$ Bragg mirrors and of $\mathrm{CdSe} / \mathrm{ZnS}$ quantum dots embedded localized in an array of pillar in the middle of the cavity, where the electromagnetic field of the resonant mode is maximum; (b) overlap of the reflectivity and the PL spectra collected from the top of the implemented device at room temperature. (For interpretation of the references to colour in this figure legend, the reader is referred to the web version of this article.)

obtaining perfectly flat mirrors, allowing a preferential field penetration along the vertical direction into the DBR layers [21]. An additional $\mathrm{SiO}_{2}$ layer was successively deposited on the surface and the planar microcavity was then completed by depositing the top dielectric Bragg mirror consisting of eight pairs of alternating $\mathrm{TiO}_{2}$ and $\mathrm{SiO}_{2}$ layers (see the sketch in Fig. 2(a)). The top and bottom $\mathrm{SiO}_{2}$ layers embedding the planarized array of pillars were designed in order to position the pillars in the middle of a $\lambda / 2$ cavity, where the electromagnetic field of the resonant mode is maximum.

\section{Results and discussion}

To investigate single-photon emission, we used a confocal microscope coupled to a standard high sensitivity Hanbury-Brown and Twiss setup. The array of nanoemitters showed different optical properties depending on the different dimensions of the pillars. Structures having a diameter above $100 \mathrm{~nm}$ presented a fluorescence typical of an ensemble of nanocrystals with a FWHM of about $30 \mathrm{~nm}$ (not shown here), whilst single-dot emission could be collected from the smallest ones, whose antibunching profile [22] (Fig. 1(b)) shows that the recorded signal from room temperature measurements arises from a single nanocrystal. Further confirmation is provided by the measured lifetime, obtained by fitting the anti-bunching trace to the function $I(t)=1-a \cdot \mathrm{e}^{-t / \tau}$ where $I(t)$ is the anti-bunching signal intensity and $\tau$ represents the single-dot decay time. We found $\tau=23 \pm 1 \mathrm{~ns}$, according with the typical lifetime for single CdSe/ZnS QDs reported in the literature [23].

Fig. 2(b) shows the overlap of the reflectivity and the PL spectra collected from the top of the implemented device at room temperature. The microcavity was designed to resonate at a wavelength of $615 \mathrm{~nm}$, as confirmed by the theoretical reflectance spectrum (red line) showing a cavity dip with a full width at half-maximum of $0.285 \mathrm{~nm}$. This results in a microcavity quality factor Q of 2140 . The PL spectrum (black line) was collected from a $500 \mathrm{~nm}$ wide pillar embedding an ensemble of CdSe/ZnS quantum dots. The cavity modulation effect on the spontaneous emission is clearly demonstrated by the narrowing of the PL linewidth down to $0.73 \mathrm{~nm}$. The decrease of the measured Q-factor could be attributed to the increased optical losses in the cavity, due to the absorption and/or scattering of the nanoclusters. 


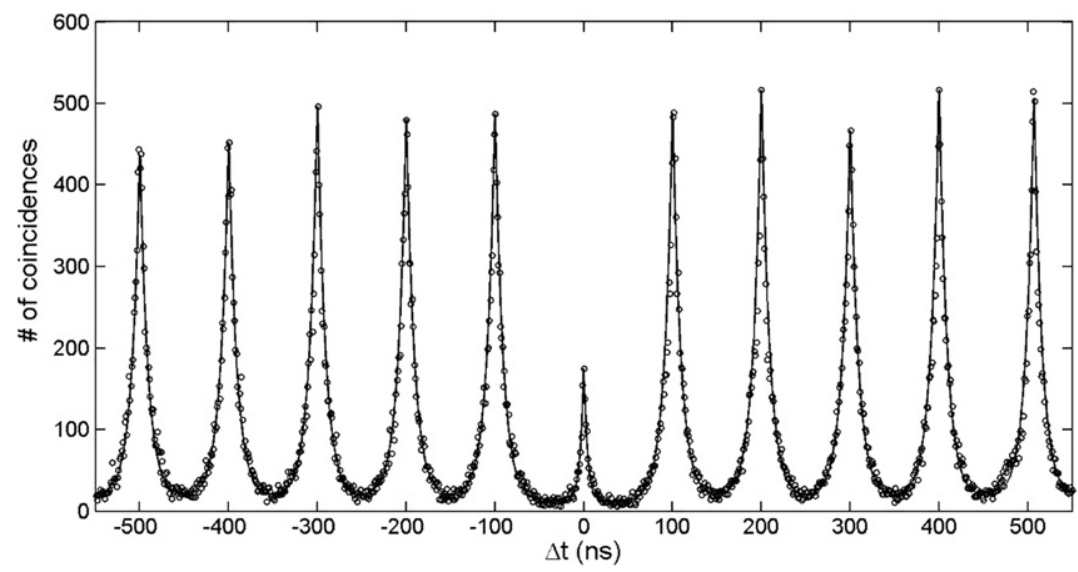

Fig. 3. Histogram of coincidence counts of single-QD fluorescence enclosed in a microcavity; the black solid line is a fit of the data, yielding a $\sim 9$ ns excited-state lifetime for the nanocrystal.

Microphotoluminescence analyses were also performed on the smaller pillars of the microcavity. In these structures, the presence of a localized single emitter is demonstrated by the histogram of the coincidences (Fig. 3) which reveals an anti-bunching behaviour at zero delay. This proves singlephoton emission at room temperature from colloidal nanocrystals embedded in a vertical microcavity. The black solid line represents a fit yielding a 9 ns excited-state lifetime for localized nanocrystal. The measured shortening of the spontaneous emission lifetime corresponds to the modification of the radiative lifetime due to the wavelength-sized cavity.

\section{Conclusions}

We proposed an approach for the fabrication of SPS based on colloidal nanocrystals, thus overcoming the limitation in the operating temperature shown by epitaxial quantum dot sources. Moreover, the structure realized does not require any kind of etching to obtain three-dimensional optical confinement; lateral confinement of the active region can be easily achieved thanks to the localization technique based on high resolution lithography. In fact, the nanometer scale control of electron beam lithography allows one to isolate pillars containing nanoemitters with arbitrarily chosen size, while the surrounding planarizing material can be chosen with a lower refractive index, thus providing lateral confinement. This strategy straightforwardly leads to the realization of mechanically robust and electrically injected vertical-emitting arrays of SPS on a single chip, appealing sources for fibre-based secure communications.

\section{Acknowledgements}

The authors gratefully acknowledge Gianmichele Epifani and Paolo Cazzato for their expert technical help.

\section{References}

[1] C. Kurtsiefer, S. Mayer, P. Zarda, H. Weinfurter, Phys. Rev. Lett. 85 (2000) 290.

[2] C. Brunel, B. Lounis, P. Tamarat, M. Orrit, Phys. Rev. Lett. 83 (1999) 2722.

[3] M. Hennrich, T. Legero, A. Kuhn, G. Rempe, New J. Phys. 6 (2004) 86.

[4] M. Pelton, C. Santori, J. Vučković, B. Zhang, G.S. Solomon, J. Plant, Y. Yamamoto, Phys. Rev. Lett. 89 (2002) 233602.

[5] K. Hennessy, A. Badolato, M. Winger, D. Gerace, M. Atatüre, S. Gulde, S. Fält, E.L. Hu, A. Imamoğlu, Nature 445 (2007) 896.

[6] S. Kako, C. Santori, K. Hoshino, S. Götzinger, Y. Yamamoto, Y. Arakawa, Nature Materials 5 (2006) 887.

[7] V.L. Colvin, M.C. Schlamp, A.P. Alivisatos, Nature 370 (1994) 354.

[8] V.I. Klimov, S.A. Ivanov, J. Nanda, M. Achermann, I. Bezel, J.A. McGuire, A. Piryatinski, Nature 447 (2007) 441-446. 
[9] L. Martiradonna, L. Carbone, A. Tandaechanurat, M. Kitamura, S. Iwamoto, L. Manna, M. De Vittorio, R. Cingolani, Y. Arakawa, Nano Lett. 8 (1) (2008) 260.

[10] X. Brokmann, L. Coolen, M. Dahan, J.-P. Hermier, Phys. Rev. Lett. 93 (2004) 107403.

[11] C.B. Murray, D.J. Norris, M.G. Bawendi, J. Am. Chem. Soc. 115 (1993) 8706.

[12] S. Hohng, T. Ha, J. Am. Chem. Soc. 126 (2004) 1324.

[13] X. Brokmann, L. Coolen, J.-P. Hermier, M. Dahan, Chem. Phys. 318 (2005) 91.

[14] P.P. Pompa, L. Martiradonna, A. Della Torre, F. Della Sala, L. Manna, M. De Vittorio, F. Calabi, R. Cingolani, R. Rinaldi, Nature Nanotech. 1 (2006) 126.

[15] R.G. Neuhauser, K.T. Shimizu, W.K. Woo, S.A. Empedocles, M.G. Bawendi, Phys. Rev. Lett. 85 (2000) 3301.

[16] G. Schlegel, J. Bohnenberger, I. Potapova, A. Mews, Phys. Rev. Lett. 88 (2002) 137401.

[17] E.M. Purcell, Phys. Rev. 69 (1946) 681.

[18] M.V. Artemyev, U. Woggon, R. Wannemacher, H. Jaschinski, W. Langbein, Nano Lett. 1 (14) (2001) 309.

[19] H. Namatsu, T. Yamaguchi, M. Nagase, K. Yamazaki, K. Kurihara, Microelectron. Eng. 41 (1998) 331.

[20] L. Martiradonna, T. Stomeo, M. De Giorgi, R. Cingolani, M. De Vittorio, Microelectron. Eng. 83 (2006) 1478.

[21] T. Plakhotnik, Optics Express 13 (2005) 3049.

[22] G. Messin, J.-P. Hermier, E. Giacobino, P. Desbiolles, M. Dahan, Opt. Lett. 26 (2001) 1891.

[23] B.R. Fisher, H.J. Eisler, N.E. Stott, M.G. Bawendi, J. Phys. Chem. B 108 (2004) 143. 